\title{
Caracterización de aislamientos de Pseudomonas aeruginosa productores de carbapenemasas de siete departamentos de Colombia
}

\author{
Sandra Yamile Saavedra, Carolina Duarte, María Nilse González, María Elena Realpe \\ Grupo de Microbiología, Dirección de Redes en Salud Pública, Subdirección Laboratorio Nacional de Referencia, \\ Instituto Nacional de Salud, Bogotá, D.C., Colombia
}

Introducción. Pseudomonas aeruginosa es un patógeno oportunista causante de múltiples infecciones en pacientes hospitalizados. Este microorganismo desarrolla fácilmente resistencia a diversos antimicrobianos, incluidos los carbapenémicos, considerados como la última opción terapéutica frente a estas infecciones. En $P$. aeruginosa la resistencia a los carbapenémicos está mediada por diferentes mecanismos: carbapenemasas de clases B (MBL) y A, alteraciones en la expresión de OprD e hiperexpresión de las bombas de expulsión Mex.

Objetivo. Describir la presencia de carbapenemasas en aislamientos de $P$. aeruginosa resistentes a carbapenémicos procedentes de siete departamentos.

Materiales y métodos. De septiembre de 2012 a marzo de 2013 se recibieron 57 aislamientos de $P$. aeruginosa en el Grupo de Microbiología del Instituto Nacional de Salud. Su identificación y perfil de sensibilidad se confirmó por Vitek $2^{\circledR}$ y técnica de difusión de disco (Kirby-Bauer) y los resultados se interpretaron según las guías del Clinical and Laboratory Standards Institute del 2013. Las carbapenemasas se detectaron fenotípicamente con el test modificado de Hodge y la prueba de sinergia para MBL usando los inhibidores EDTA-SMA, y genotípicamente con PCR convencional para los genes blaKPC, blaVIM, blalMP y blaNDM.

Resultados. De los 57 aislamientos remitidos, dos fueron sensibles a carbapenémicos y, de los 55 restantes, en 43 se confirmó la presencia de carbapenemasas; el mayor porcentaje de sensibilidad se presentó frente a la colistina $(96,4 \% ; n=53)$. Los 43 aislamientos productores de carbapenemasas presentaron perfil de multirresistencia: $76,7 \%$ fue positivo con el test modificado de Hodge y $79,1 \%$ presentó sinergia con MBL. Treinta y tres aislamientos fueron positivos para blaVIM, nueve para blaKPC y un aislamiento fue productor tanto de carbapenemasas KPC como de VIM. Ningún aislamiento amplificó para blalMP y blaNDM.

Conclusiones. La carbapenemasa más frecuente fue la VIM seguida de la KPC en una relación aproximada de 3:1.

Palabras clave: Pseudomonas aeruginosa, carbapenémicos, farmacorresistencia microbiana. doi: http://dx.doi.org/10.7705/biomedica.v34i0.1685

Characterization of isolates of carbapenemase-producing Pseudomonas aeruginosa from seven Colombian provinces

Introduction: Pseudomonas aeruginosa is an opportunistic pathogen that causes multiple infections in hospitalized patients. This microorganism has developed resistance to several antimicrobial agents, including carbapenems, which are considered to be the last therapeutic option against these infections. Carbapenem resistance of $P$. aeruginosa is mediated by different mechanisms: Carbapenemases class $B(M B L)$ and $A$, alterations in the OprD expression and overexpression of the Mex efflux pump.

Objective: To describe the presence of carbapenemases in $P$. aeruginosa isolates from seven Colombian provinces.

Materials and methods: A total of 57 P. aeruginosa isolates were collected between September 2012 and March 2013 from national surveillance in Colombia and were sent to the Grupo de Microbiología at the Instituto Nacional de Salud (INS) for evaluation. lidentification and antimicrobial susceptibility were confirmed through automated method (Vitek $\left.{ }^{\circledR} 2\right)$ and disk diffusion (Kirby-Bauer) according to the Clinical and Laboratory Standards Institute, CLSI, 2013. Phenotypic and genotypic confirmation was determined using the modified Hodge test (MHT), a synergism test using imipenem, EDTA-SMA and meropenem, and conventional PCR to detect the blaKPC, blaVIM, blalMP and blaNDM genes.

Results: Of the 57 isolates, two showed sensitivity to carbapenems. Forty-three isolates were positive

Contribución de los autores:

María Nilse González, Carolina Valderrama y Sandra Yamile Saavedra: caracterización de los aislamientos.

Todos los autores participaron en la escritura, revisión y corrección del manuscrito. 
for carbapenemases with a high percentage of sensitivity to colistin $(76.4 \%, \mathrm{n}=42)$. The 43 isolates producing carbapenemases showed multiple drug resistance: $72.1 \%$ were positive in the MHT and $79.1 \%$ showed MBL synergism. PCR amplification confirmed that 33 isolates were positive for blaVIM, nine were positive for blaKPC and one isolate expressed both KPC and VIM carbapenemases. No isolates showed amplified products with blalMP and blaNDM primers.

Conclusions: The most frequent carbapenemase was VIM, followed by KPC in an approximate ratio of 3:1.

Key words: Pseudomonas aeruginosa, carbapenems; drug resistance, microbial.

doi: http://dx.doi.org/10.7705/biomedica.v34i0.1685

Pseudomonas aeruginosa es un patógeno oportunista de gran relevancia clínica que se encuentra entre los cinco gérmenes más aislados en hospitales de Latinoamérica, incluido Colombia $(1,2)$. Este microorganismo es causante de múltiples infecciones, especialmente en unidades de cuidados intensivos, en pacientes inmunocomprometidos y en pacientes con fibrosis quística $(1,3,4)$. Al tener mínimos requerimientos nutricionales, este microorganismo puede sobrevivir a diversas condiciones físicas del ambiente hospitalario, y se ha podido aislar en superficies, equipo hospitalario, medicamentos, antisépticos, jabones y diversas soluciones (3).

El tratamiento de infecciones causadas por $P$. aeruginosa es difícil por la facilidad de este microorganismo para desarrollar resistencia a diferentes antimicrobianos, ya sea de manera intrínseca (mediante la alteración de la permeabilidad, las bombas de expulsión y la mutación en la betalactamasa AmpC cromosómica), por adquisición de determinantes de resistencia (carbapenemasas) o por la presencia de ambos factores (3-5). Aunque los carbapenémicos se consideran una de las últimas opciones terapéuticas para infecciones por $P$. aeruginosa multirresistentes, en los últimos años se ha incrementado la circulación de cepas resistentes a estos antimicrobianos a nivel mundial (5-9). En este microorganismo la resistencia a carbapenémicos está asociada con: (i) presencia de carbapenemasas de las clases A (KPC, GES, BIC) y B (VIM, IMP, SPM, NDM, DIM, AIM, FIM) y, en menor proporción, D (OXA-40); (ii) pérdida o reducción de la expresión de la porina OprD; (iii) hiperexpresión de los sistemas de expulsión

\section{Correspondencia:}

Sandra Yamile Saavedra, Grupo de Microbiología, Dirección de Redes en Salud Pública, Subdirección Laboratorio Nacional de Referencia, Instituto Nacional de Salud, Calle $26 N^{\circ}$ 51-20, Bogotá, D.C., Colombia.

Teléfono: (571) 2207700 , extensión 1421

sysaavedrar@gmail.com

Recibido: 05/06/13; aceptado: 14/11/13
Mex (principalmente MexAB-OprM y, en menor proporción, MexCD-OprJ y Mex XY-OprM), y (iv) sinergia entre alteraciones de permeabilidad y expresión de bombas de expulsión con presencia de hiperexpresión de la enzima AmpC cromosómica o sin ella $(3-5,10)$.

En este estudio se describe la presencia de carbapenemasas con métodos fenotípicos y genotípicos en aislamientos de $P$. aeruginosa recuperados de siete departamentos en el contexto del Programa de Vigilancia por Laboratorio de Resistencia Bacteriana de Infecciones Asociadas a la Atención en Salud del Grupo de Microbiología del Instituto Nacional de Salud seis meses después de iniciado el programa.

\section{Materiales y métodos}

Desde el año 2012, el Grupo de Microbiología de la Dirección de Redes en Salud Pública inició la capacitación de los laboratorios departamentales de salud pública y su respectiva red de laboratorios públicos y privados en temas relacionados con la resistencia antimicrobiana en bacterias Gram positivas y Gram negativas. En lo concerniente a enterobacterias y bacterias no fermentadoras, se capacita en la detección fenotípica de carbapenemasas de tipo serina y metaloenzimas. Hasta la fecha se han capacitado los laboratorios departamentales de salud pública de Antioquia, Atlántico, Boyacá, Cundinamarca, Santander, Norte de Santander, Valle del Cauca y a la Secretaría Distrital de Salud de Bogotá. Estos centros ya capacitados envían aislamientos sospechosos de carbapenemasas al Grupo de Microbiología para su confirmación fenotípica y genotípica.

\section{Tipo de estudio}

Se llevó a cabo un estudio descriptivo.

\section{Aislamientos y caracterización microbiológica}

Entre septiembre de 2012 y marzo de 2013 el Grupo de Microbiología recibió 57 aislamientos de $P$. aeruginosa considerados sospechosos de ser 
productores de carbapenemasas. Se confirmó la identificación y el perfil de sensibilidad de todos los aislamientos recibidos por el sistema automatizado Vitek 2 Compac® (bioMérieux, tarjeta AST-N082) y, además, se evaluó la sensibilidad de los siguientes antimicrobianos por medio de la técnica de difusión en disco (Kirby-Bauer): amicacina, aztreonam, cefepime, ceftazidima, ciprofloxacina, gentamicina, imipenem, meropenem y piperacilina-tazobactam. La interpretación de los resultados se realizó de acuerdo a los parámetros establecidos por el Clinical and Laboratory Standards Institute (CLSI) para el 2013, usando como cepa de control $P$. aeruginosa ATCC 27853 (11).

Para la detección de carbapenemasas se realizó el test modificado de Hodge utilizando como cepa indicadora Klebsiella pneumoniae ATCC 700603, como control positivo, la cepa K. pneumoniae BAA1705 (positiva para KPC) y como control negativo, la cepa K. pneumoniae BAA-1706 (12); para la detección de las carbapenemasas de clase $B$ (metalobetalactamasa, MBL) se realizó la prueba de sinergia usando discos de imipenem $(10 \mu \mathrm{g})$, inhibidores EDTA-SMA (750 $\mu \mathrm{g}$ de ácido etilendiamino-tetraacético más $2 \mathrm{mg}$ de mercaptoacetato de sodio) (13) y meropenem (10 $\mu \mathrm{g})$, ubicando los discos a una distancia de $15 \mathrm{~mm}$ entre centro y centro según las recomendaciones del Instituto Nacional de Enfermedades Infecciosas "Dr. Carlos G. Malbrán" que actúa como Centro de Referencia Regional de la Organización Panamericana de la Salud. El control positivo usado en esta prueba fue la cepa K. pneumoniae BAA- 2146 (positiva para NDM).

\section{Detección genotípica de carbapenemasas}

Todos los aislamientos con sensibilidad intermedia a, por lo menos, uno de los carbapenémicos evaluados, o positivo con el test modificado de Hodge o en la prueba para metalobetalactamasas, se sometieron a la detección de carbapenemasas por PCR convencional. En los aislamientos, la extracción de ADN se realizó por lisis celular mediante ebullición durante 10 minutos. A partir del ADN total se analizó la presencia de los genes codificadores de las carbapenemasas KPC, IMP, VIM y NDM utilizando los iniciadores descritos en el cuadro 1.

La reacción de amplificación se llevó a cabo en una mezcla que contenía $0,2 \mathrm{mM}$ de dNTPs, $1,5 \mathrm{mM}$ de $\mathrm{MgCl} 2,0,4 \mu \mathrm{M}$ de cada iniciador (excepto los iniciadores para IMP que se usaron con una concentración de 0,5 $\mu \mathrm{M}$ ), y $0,06 \mathrm{U} / \mu \mathrm{l}$ de ADN polimerasa Taq (Invitrogen ${ }^{\circledR}$ ). La reacción de amplificación se realizó bajo las siguientes condiciones: $95^{\circ} \mathrm{C}$ por 5 minutos, 35 ciclos a $95^{\circ} \mathrm{C}$ por 30 segundos, $\mathrm{KPC}\left(62^{\circ} \mathrm{C}\right)$, NDM $\left(63^{\circ} \mathrm{C}\right)$, VIM $\left(60^{\circ} \mathrm{C}\right)$ e IMP $\left(61^{\circ} \mathrm{C}\right)$ por 45 segundos a $72{ }^{\circ} \mathrm{C}$ por un minuto y una extensión final a $72{ }^{\circ} \mathrm{C}$ por siete minutos en un termociclador T100 Thermal Cycler ${ }^{\circledast}$ de Bio-Rad. Los controles positivos utilizados fueron K. pneumoniae BAA-1705 (positiva para KPC), K. pneumoniae BAA-2146 (positiva para NDM), P. aeruginosa VIM-2 (Universidad Nacional de Colombia) y Enterobacter cloacae OPS-166 (positiva para IMP).

\section{Resultados}

\section{Caracterización microbiológica}

De los 57 aislamientos recibidos, dos fueron sensibles a carbapenémicos y, de los 55 restantes, en 43 se confirmó la presencia de carbapenemasas. Según el perfil de sensibilidad, el mayor porcentaje se presentó frente a la colistina $(96,4 \% ; n=53)$; los 43 aislamientos productores de carbapenemasas presentaron perfil de multirresistencia (resistentes a tres familias de antimicrobianos) comparados con los aislamientos no productores de carbapenemasas (cuadro 2). Los aislamientos productores de carbapenemasas KPC presentaron un perfil de resistencia similar, probablemente asociado al hecho de que dichos aislamientos provenían de un solo departamento (cuadro 3), mientras que los

Cuadro 1. Lista de iniciadores evaluados en el estudio

\begin{tabular}{llcc}
\hline Iniciador & \multicolumn{1}{c}{ Secuencia 5' $-\mathbf{3}^{\prime}$} & Tamaño $(\mathbf{p b})$ & Referencia \\
\hline KPC-F & ATGTCACTGTATCGCCGTCT & 893 & 14 \\
KPC-R & TTTTCAGAGCCTTACTGCCC & & 188 \\
IMPgen-F1 & GAATAG(A/G)(A/G)TGGCTTAA(C/T)TCTC & & 15 \\
IMPgen-R1 & CCAAAC(C/T)ACTA(G/C)GTTATC & 382 & 15 \\
VIMgen-F2 & GTTTGGTCGCATATCGCAAC & & \\
VIMgen-R2 & AATGCGCAGCACCAGGATAG & 661 & 16 \\
NDM-F & GGTGCATGCCCGGTGAAATC & \\
NDM-R & ATGCTGGCCTTGGGGAACG &
\end{tabular}


Cuadro 2. Sensibilidad antimicrobiana de aislamientos de Pseudomonas aeruginosa resistentes a carbapenémicos

\begin{tabular}{|c|c|c|c|c|c|c|c|c|c|c|c|c|c|c|c|}
\hline \multirow{3}{*}{ Antimicrobiano } & \multicolumn{15}{|c|}{ Aislamientos de $P$. aeruginosa } \\
\hline & \multicolumn{3}{|c|}{ Total $(n=55)$} & \multicolumn{3}{|c|}{$\begin{array}{l}\text { No productores de } \\
\text { carbapenemasas } \\
\qquad(n=12)\end{array}$} & \multicolumn{3}{|c|}{$K P C+\operatorname{VIM}(n=1)$} & \multicolumn{3}{|c|}{$\operatorname{KPC}(n=9)$} & \multicolumn{3}{|c|}{ VIM $(n=33)$} \\
\hline & $\mathbf{S}(\%)$ & I (\%) & $\mathbf{R}(\%)$ & $\mathrm{S}(\%)$ & $\mathrm{I}(\%)$ & $\mathbf{R}(\%)$ & $\mathbf{S}(\%)$ & I (\%) & $\mathbf{R}(\%)$ & S (\%) & I (\%) & $\mathbf{R}(\%)$ & $\mathbf{S}(\%)$ & I (\%) & $\mathbf{R}(\%)$ \\
\hline Imipenem & 3,6 & 1,8 & 94,6 & 16,7 & 0 & 83,3 & 0 & 0 & 100 & 0 & 0 & 100 & 0 & 0 & 100 \\
\hline Meropenem & 0 & 3,6 & 96,4 & 0 & 16,7 & 83,3 & 0 & 0 & 100 & 0 & 0 & 100 & 0 & 0 & 100 \\
\hline Ceftazidima & 20 & 18,2 & 61,8 & 83,3 & 0 & 16,7 & 0 & 0 & 100 & 0 & 0 & 100 & 3 & 30,3 & 66,7 \\
\hline Aztreonam* & 21,8 & 30,9 & 47,3 & 25 & 41,7 & 33,3 & 0 & 0 & 100 & 0 & 0 & 100 & 27,2 & 36,4 & 36,4 \\
\hline Cefepime & 32,7 & 21,8 & 45,5 & 83,4 & 8,3 & 8,3 & 0 & 0 & 100 & 0 & 0 & 100 & 24,2 & 33,3 & 42,5 \\
\hline $\begin{array}{l}\text { Piperacilina- } \\
\text { tazobactam }\end{array}$ & 14,5 & 36,4 & 49,1 & 41,7 & 41,7 & 16,6 & 0 & 0 & 100 & 0 & 0 & 100 & 9 & 45,5 & 45,5 \\
\hline Amicacina & 34,6 & 3,6 & 61,8 & 66,7 & 0 & 33,3 & 0 & 0 & 100 & 88,9 & 0 & 11,1 & 9 & 6,1 & 84,9 \\
\hline Gentamicina & 25,4 & 7,3 & 67,3 & 66,7 & 8,3 & 25 & 0 & 0 & 100 & 0 & 0 & 100 & 18,2 & 9 & 72,8 \\
\hline Ciprofloxacina & 14,5 & 5,5 & 80 & 50 & 8,3 & 41,7 & 0 & 0 & 100 & 0 & 0 & 100 & 6,1 & 6,1 & 87,8 \\
\hline Colistina** & 96,4 & 1,8 & 1,8 & 100 & 0 & 0 & 100 & 0 & 0 & 100 & 0 & 0 & 94 & 3 & 3 \\
\hline
\end{tabular}

S: aislamientos sensibles; I: aislamientos con sensibilidad intermedia; R: aislamientos resistentes

*Antimicrobiano evaluado únicamente por Kirby Bauer; ${ }^{* *}$ Antimicrobiano evaluado únicamente por sistema automatizado Vitek

aislamientos productores de carbapenemasas VIM presentaron un perfil de sensibilidad más variado (cuadro 3).

En cuanto a las pruebas fenotípicas para la detección de carbapenemasas, se observó que de los 43 aislamientos productores de carbapenemasas, $76,7 \%(n=33)$ fue positivo con el test modificado de Hodge y $79,1 \%(n=34)$ lo fue con los inhibidores EDTA-SMA; todos los aislamientos productores de KPC $(n=9)$ fueron positivos en el test modificado de Hodge y negativos con EDTA-SMA (cuadro 3); de los 33 aislamientos productores de VIM, solo $69,7 \%(n=23)$ fue positivo en el test modificado de Hodge y todos fueron positivos para la presencia de MBL con EDTA-SMA (cuadro 3), excepto dos de ellos (RB 245 y RB 286), que fueron negativos en la prueba con EDTA-SMA, pero positivos con las tiras de la prueba de epsilometría (E-test) para MBL (imipenem-imipenem+EDTA). El aislamiento coproductor de KPC y VIM fue positivo para las dos pruebas fenotípicas realizadas para carbapenemasas (cuadro 3). Ningún aislamiento negativo para carbapenemasas dio resultado positivo en el test modificado de Hodge ni en la prueba de sinergia con EDTA-SMA.

\section{Detección genotípica de carbapenemasas}

De los 43 aislamientos positivos para carbapenemasas, 33 fueron positivos para el gen blaVIM, nueve para el gen blaKPC, y en un aislamiento se detectó producción de las carbapenemasas KPC y VIM. Ningún aislamiento amplificó para las carbapenemasas IMP y NDM (cuadro 3).

\section{Discusión}

En este estudio se observó que la carbapenemasa más frecuente en los aislamientos de $P$. aeruginosa fue la VIM, seguida de la KPC, con una relación aproximada de 3:1. A nivel mundial se observa un comportamiento similar, encontrándose que en $P$. aeruginosa las carbapenemasas más detectadas son las del tipo de las metalobetalactamasas $(5,17)$. Ambas enzimas han sido detectadas en aislamientos de $P$. aeruginosa de Colombia: la primera detectada fue la VIM-8, hallada en Cali en muestras aisladas desde 1999 (8); la segunda MBL fue la VIM-2, la cual se encontró diseminada en seis departamentos (18). Posteriormente se realizó el primer hallazgo a nivel mundial de KPC-2 en $P$. aeruginosa en aislamientos provenientes de Medellín (9) que, hasta la fecha, se han detectado tambien en más de cinco departamentos (19).

Los aislamientos de $P$. aeruginosa de tipo VIM se detectaron en los siete departamentos del estudio; $94.1 \%$ de estos aislamientos presentó multirresistencia, pero con un perfil de sensibilidad variado. Es motivo de gran preocupación que la mayoría de los aislamientos analizados presentó un perfil de multirresistencia, especialmente aquellos resistentes a todos los antimicrobianos evaluados y los resistentes a colistina, pues aunque este viejo antimicrobiano con actividad contra pseudomonas se había dejado de usar por su toxicidad, ante el surgimiento de aislamientos multirresistentes hoy se le considera una de las opciones de tratamiento contra estas cepas (4). 
Cuadro 3. Origen y descripción fenotípica y genotípica de aislamientos de Pseudomonas aeruginosa resistentes a carbapenémicos

\begin{tabular}{|c|c|c|c|c|c|c|c|c|c|c|}
\hline \multirow[t]{2}{*}{ Código } & \multirow[t]{2}{*}{ Muestra } & \multirow[t]{2}{*}{ Departamento } & \multicolumn{4}{|c|}{ Caracterización microbiológica } & \multirow[t]{2}{*}{ blaKPC } & \multirow[t]{2}{*}{ blaVIM } & \multirow[t]{2}{*}{ blalMP } & \multirow[t]{2}{*}{ blaNDM } \\
\hline & & & $\begin{array}{c}\text { IMP } \\
\mathrm{CIM} \mu \mathrm{g} / \mathrm{ml}\end{array}$ & $\begin{array}{c}\text { MEM } \\
\text { CIM } \mu \mathrm{g} / \mathrm{ml}\end{array}$ & TMH & $\begin{array}{l}\text { EDTA- } \\
\text { SMA }\end{array}$ & & & & \\
\hline RB 44 & Lavado broncoalveolar & Valle del Cauca & $\geq 16$ & $\geq 16$ & + & - & + & - & - & - \\
\hline RB 54 & Orina & Antioquia & $\geq 16$ & $\geq 16$ & + & + & - & + & - & - \\
\hline RB 55 & Orina & Santander & $\geq 16$ & $\geq 16$ & + & + & - & + & - & - \\
\hline RB 56 & Secreción orotraqueal & Santander & $\geq 16$ & 4 & - & - & - & - & - & - \\
\hline RB 58 & Orina & Cauca & $\geq 16$ & $\geq 16$ & + & + & - & + & - & - \\
\hline RB 61 & Secreción orotraqueal & Cauca & $\geq 16$ & 8 & - & + & - & + & - & - \\
\hline RB 63 & Sin dato & Cundinamarca & $\geq 16$ & $\geq 16$ & + & + & - & + & - & - \\
\hline RB 64 & Sin dato & Cundinamarca & $\geq 16$ & $\geq 16$ & + & + & - & + & - & - \\
\hline RB 74 & Sangre & Cundinamarca & $\geq 16$ & $\geq 16$ & - & + & - & + & - & - \\
\hline RB 77 & Secreción orotraqueal & Santander & $\geq 16$ & $\geq 16$ & + & + & - & + & - & - \\
\hline RB 78 & Secreción orotraqueal & Santander & $\geq 16$ & $\geq 16$ & - & - & - & - & - & - \\
\hline RB 80 & Secreción pancreática & Antioquia & $\geq 16$ & $\geq 16$ & + & + & - & + & - & - \\
\hline RB 83 & Orina & Cundinamarca & $\geq 16$ & $\geq 16$ & - & + & - & + & - & - \\
\hline RB 84 & Sin dato & Cundinamarca & $\geq 16$ & $\geq 16$ & - & + & - & + & - & - \\
\hline RB 85 & Orina & Cundinamarca & $\geq 16$ & 8 & + & + & - & + & - & - \\
\hline RB 86 & Secreción orotraqueal & Santander & $\geq 16$ & 8 & - & - & - & - & - & - \\
\hline RB 94 & Secreción orotraqueal & Boyacá & $\leq 1$ & 8 & - & - & - & - & - & - \\
\hline RB 100 & Secreción orotraqueal & Cundinamarca & $\geq 16$ & $\geq 16$ & + & + & - & + & - & - \\
\hline RB 105 & Sin dato & Antioquia & $\geq 16$ & $\geq 16$ & - & - & - & - & - & - \\
\hline RB 108 & Líquido cefalorraquídeo & Meta & $\geq 16$ & $\geq 16$ & + & + & - & + & - & - \\
\hline RB 112 & Sangre & Santander & $\geq 16$ & $\geq 16$ & + & + & - & + & - & - \\
\hline RB 113 & Orina & Santander & $\geq 16$ & $\geq 16$ & + & + & - & + & - & - \\
\hline RB 114 & Orina & Norte de Santander & $\geq 16$ & $\geq 16$ & + & + & - & + & - & - \\
\hline RB 136 & Secreción bronquial & Norte de Santander & $\geq 16$ & $\geq 16$ & + & + & - & + & - & - \\
\hline RB 149 & Catéter & Santander & $\geq 16$ & $\geq 16$ & - & + & - & + & - & - \\
\hline RB 158-1 & Sangre & Valle del Cauca & $\geq 16$ & $\geq 16$ & - & - & - & - & - & - \\
\hline RB 162 & Líquido abdominal & Cundinamarca & $\geq 16$ & $\geq 16$ & + & + & - & + & - & - \\
\hline RB 183-1 & $\begin{array}{l}\text { Secreción de miembro } \\
\text { inferior derecho }\end{array}$ & Valle del Cauca & $\geq 16$ & $\geq 16$ & - & + & - & + & - & - \\
\hline RB 195 & Secreción orotraqueal & Valle del Cauca & $\geq 16$ & $\geq 16$ & + & - & + & - & - & - \\
\hline RB 203 & Herida en tórax & Boyacá & $\geq 16$ & $\geq 16$ & - & - & - & - & - & - \\
\hline RB 208 & Peritoneo & Santander & $\geq 16$ & $\geq 16$ & + & + & - & + & - & - \\
\hline RB 210 & Orina & Santander & $\geq 16$ & $\geq 16$ & + & + & - & + & - & - \\
\hline RB 221 & Orina & Valle del Cauca & $\geq 16$ & $\geq 16$ & + & - & + & - & - & - \\
\hline RB 223 & Líquido cefalorraquídeo & Valle del Cauca & $\geq 16$ & $\geq 16$ & + & - & + & - & - & - \\
\hline RB 224 & Orina & Valle del Cauca & $\geq 16$ & $\geq 16$ & + & - & + & - & - & - \\
\hline RB 225 & Sangre & Valle del Cauca & $\geq 16$ & $\geq 16$ & - & + & - & + & - & - \\
\hline RB 226 & Herida quirúrgica & Valle del Cauca & $\geq 16$ & $\geq 16$ & + & - & + & - & - & - \\
\hline RB 228 & Secreción rodilla & Boyacá & $\geq 16$ & $\geq 16$ & - & - & - & - & - & - \\
\hline RB 229 & Sangre & Boyacá & 2 & 4 & - & - & - & - & - & - \\
\hline RB 233 & Secreción, úlcera sacra & Norte de Santander & $\geq 16$ & $\geq 16$ & + & + & - & + & - & - \\
\hline RB 234 & Secreción, pierna derecha & Norte de Santander & $\geq 16$ & 8 & + & + & - & + & - & - \\
\hline RB 235 & Orina & Norte de Santander & $\geq 16$ & $\geq 16$ & - & - & - & - & - & - \\
\hline RB 243 & Líquido pleural & Cundinamarca & $\geq 16$ & $\geq 16$ & - & + & - & + & - & - \\
\hline RB 245 & Secreción, pierna izquierda & Valle del Cauca & $\geq 16$ & $\geq 16$ & - & $+^{\star}$ & - & + & - & - \\
\hline RB 247 & Lecho ventricular & Valle del Cauca & $\geq 16$ & $\geq 16$ & + & - & + & - & - & - \\
\hline RB 248 & Miembro inferior derecho & Valle del Cauca & $\geq 16$ & $\geq 16$ & + & + & - & + & - & - \\
\hline RB 249 & Sangre & Valle del Cauca & $\geq 16$ & $\geq 16$ & + & + & - & + & - & - \\
\hline RB 251 & Secreción traqueal & Valle del Cauca & $\geq 16$ & $\geq 16$ & + & - & + & - & - & - \\
\hline RB 252 & Orina & Valle del Cauca & $\geq 16$ & $\geq 16$ & + & - & + & - & - & - \\
\hline RB 255 & Orina & Santander & $\geq 16$ & $\geq 16$ & + & + & - & + & - & - \\
\hline RB 257 & Sangre & Santander & $\geq 16$ & $\geq 16$ & - & + & - & + & - & - \\
\hline RB 282 & Sin dato & Antioquia & $\geq 16$ & 8 & - & - & - & - & - & - \\
\hline RB 286 & Sin dato & Antioquia & $\geq 16$ & $\geq 16$ & + & $+^{\star}$ & - & + & - & - \\
\hline RB 287 & Sin dato & Antioquia & $\geq 16$ & $\geq 16$ & - & - & - & - & - & - \\
\hline RB 290 & Secreción, pierna & Norte de Santander & $\geq 16$ & 8 & + & + & + & + & - & - \\
\hline
\end{tabular}

CIM: concentración inhibitoria mínima; THM: test modificado de Hodge

IPM: imipenem; MEM: meropenem

* Prueba para carbapenemasas de clases B positivas para MBL realizada con tiras de E-test impregnadas con imipenem-imipenem+EDTA 
Dos de los 34 aislamientos positivos para el gen blaVIM fueron negativos en la detección fenotípica con EDTA-SMA, pero positivos para MBL con las tiras de E-test; este fenómeno de resultado falso negativo al emplear como inhibidores EDTA y SMA fue descrito por Yong, et al. (20), quienes observaron la baja sensibilidad de esta prueba en la detección de aislamientos positivos para MBL frente a otros métodos que usan EDTA y ácido dipicolínico (DPA) como inhibidores; sin embargo, los ensayos con EDTA-SMA y DPA presentan una alta especificidad comparados con el método de EDTA, el cual presenta una especificidad de $11,4 \%$ (20). Aunque en nuestro estudio este falso negativo se presentó solo en dos aislamientos, es necesario estudiar las características de permeabilidad de estos inhibidores con el fin de establecer la posible causa del fenómeno.

En cuanto al test modificado de Hodge, autores como Carvalhaes, et al., y Bonnin, et al., han descrito la baja sensibilidad de esta prueba en la detección de carbapenemasas MBL $(21,22)$, fenómeno que también se observó en nuestro estudio, en el que aproximadamente $30 \%$ de los aislamientos positivos para VIM fue negativo en el test modificado de Hodge. Lo contrario se observó en los aislamientos de $P$. aeruginosa positivos para KPC, ya que $100 \%$ fue positivo en el test modificado de Hodge; sin embargo, se debe ser cuidadoso en el momento de la lectura para evitar dar resultados falsos positivos o falsos negativos.

Los nueve aislamientos de $P$. aeruginosa positivos para el gen KPC fueron enviados del departamento del Valle del Cauca. Todos estos aislamientos fueron sensibles a colistina, al igual que los aislamientos de $P$. aeruginosa positivos para KPC-2 reportados previamente en Colombia (19); asimismo, todos presentaron resistencia a los antimicrobianos evaluados, excepto a la amicacina y la colistina (menos el aislamiento RB 251 resistente a amicacina). La similitud en el perfil de sensibilidad de los aislamientos se relacionó con el hecho de que todos fueron enviados desde este departamento, por lo cual sería adecuado realizar un análisis de relación genética en dichos aislamientos para determinar si allí hay circulación de un genotipo específico.

Solo uno de los aislamientos analizados, remitido del departamento de Norte de Santander, presentó producción de carabapenemasas KPC y VIM (aislamiento RB 290). Una cepa con esta característica ya había sido previamente detectada en Cali (23), pero a diferencia de este aislamiento, la muestra RB 290 fue sensible únicamente a colistina y, además, fue positiva en las pruebas microbiológicas con el test modificado de Hodge y EDTA-SMA. Las cepas productoras de dos carbapenemasas representan un desafío mayor para la salud pública debido a la posibilidad de su diseminación exitosa y a la dificultad del tratamiento de infecciones causadas por estos aislamientos multirresistentes.

En conclusión, teniendo en cuenta la amplia circulación de cepas de $P$. aeruginosa portadoras de carbapenemasas de tipo KPC y VIM en nuestro medio, es necesario seguir implementando la búsqueda fenotípica de aislamientos sospechosos en los laboratorios clínicos y su confirmación en el laboratorio de referencia, pues su diseminación constituye una amenaza para la salud pública.

Este estudio describe nuestra experiencia con aislamientos de $P$. aeruginosa productores de carbapenemasas en los seis meses transcurridos desde el inicio del Programa de Vigilancia por Laboratorio de Resistencia Bacteriana de Infecciones Asociadas a la Atención en Salud a cargo del Grupo de Microbiología del Instituto Nacional de Salud.

\section{Agradecimientos}

A los laboratorios departamentales de salud pública de Antioquia, Atlántico, Boyacá, Cundinamarca, Norte de Santander, Santander y Valle del Cauca, y a su respectiva red de laboratorios públicos y privados, así como a la Secretaría Distrital de Salud de Bogotá.

\section{Conflicto de intereses}

Los autores participantes manifiestan no presentar ningún conflicto de intereses.

\section{Financiación}

El estudio fue financiado por el Instituto Nacional de Salud.

\section{Referencias}

1. Gales AC, Castanheira M, Jones RN, Sader HS. Antimicrobial resistance among Gram-negative bacilli isolated from Latin America: Results from SENTRY Antimicrobial Surveillance Program (Latin America, 20082010). Diagn Microbiol Infect Dis. 2012;73:354-60. http:// dx.doi/10.1016/j.diagmicrobio.2012.04.007

2. Villalobos Rodríguez AP, Díaz Ortega MH, Barrero Garzón LI, Rivera Vargas SM, Henríquez Iguarán DE, Villegas Botero MV, et al. Tendencias de los fenotipos de resistencia bacteriana en hospitales públicos y privados 
de alta complejidad de Colombia. Rev Panam Salud Pública. 2011:30:627-33. http://dx.doi.org/10.1590/S102049892011001200022

3. Lister PD, Wolter DJ, Hanson ND. Antibacterialresistant Pseudomonas aeruginosa: Clinical impact and complex regulation of chromosomally encoded resistance mechanisms. Clin Microbiol Rev. 2009;22:582-610. http://dx. doi/10.1128/CMR.00040-09

4. Driscoll JA, Brody SL, Kollef MH. The epidemiology, pathogenesis and treatment of Pseudomonas aeruginosa infections. Drugs. 2007;67:351-68. http://dx.doi/10.2165/ 00003495-200767030-00003

5. Nicolau CJ, Oliver A. Carbapenemasas en especies del género Pseudomonas. Enferm Infecc Microbiol Clin. 2010;28:19-28. http://dx.doi/10.1016/S0213-005X(10)70004-5

6. Santella G, Pollini S, Docquier JD, Almuzara M, Gutkind G, Rossolini G, et al. Resistencia a carbapenemes en aislamientos de Pseudomonas aeruginosa: un ejemplo de interacción entre distintos mecanismos. Rev Panam Salud Pública. 2011;30:545-8. http://dx.doi.org/10.1590/S102049892011001200008

7. Gales AC, Menezes LC, Silbert S, Sader HS. Dissemination in distinct Brazilian regions of an epidemic carbapenemresistant Pseudomonas aeruginosa producing SPM metallobeta-lactamase. J Antimicrob Chemother. 2003;52:699-702. http://dx.doi/10.1093/jac/dkg416

8. Crespo MP, Woodford N, Sinclair A, Kaufmann ME, Turton J, Glover J, et al. Outbreak of carbapenemresistant Pseudomonas aeruginosa producing VIM-8, a novel metallo-beta-lactamase, in a tertiary care center in Cali, Colombia. J Clin Microbiol. 2004;42:5094-101. http:// dx.doi/10.1128/JCM.42.11.5094-5101.2004

9. Villegas MV, Lolans K, Correa A, Kattan JN, López JA, Quinn JP. First identification of Pseudomonas aeruginosa isolates producing a KPC-type carbapenem-hydrolyzing betalactamase. Antimicrob Agents Chemother. 2007;51:1553-5. http://dx.doi:10.1128/AAC.01405-06

10. Patel G, Bonomo RA. "Stormy waters ahead": Global emergence of carbapenemases. Front Microbiol. 2013;4:48. http://dx.doi/10.3389/fmicb.2013.00048

11. Clinical and Laboratory Standards Institute. Performance Standards for Antimicrobial Susceptibility Testing: Twentieth Informational Supplement M100-S23. Wayne, PA: CLSI; 2013.

12. Pasteran F, Veliz O, Rapoport M, Guerriero L, Corso A. Sensitive and specific modified Hodge test for KPC and metallo-beta- lactamase detection in Pseudomonas aeruginosa by use of a novel indicator strain, Klebsiella pneumoniae ATCC 700603. J Clin Microbiol. 2011;49:4301-3. http://dx.doi/10.1128/JCM.05602-11

13. Lee K, Lim YS, Yong D, Yum JH, Chong Y. Evaluation of the Hodge test and the imipenem-EDTA double-disk synergy test for differentiating metallo-beta-lactamase- producing isolates of Pseudomonas spp. and Acinetobacter spp. J Clin Microbiol. 2003;41:4623-9. http://dx.doi/10.1128/ JCM.41.10.4623-4629.2003

14. Bradford PA, Bratu S, Urban C, Visalli M, Mariano N, Landman D, et al. Emergence of carbapenem-resistant Klebsiella species possessing the class A carbapenemhydrolyzing KPC-2 and inhibitor-resistant TEM-30 betalactamases in New York City. Clin Infect Dis. 2004;39:55-60. http://dx.doi/10.1086/421495

15. Mendes R, Kiyota K, Monteiro J, Castanheira M, Andrade S, Gales A, et al. Rapid detection and identification of metallo-B- lactamase-encoding genes by multiplex realtime PCR assay and melt curve analysis. J Clin Microbiol. 2007;45:544-7. http://dx.doi/10.1128/JCM.01728-06

16. Nordmann P, Poirel L, Carrër A, Toleman MA, Walsh TR. How to detect NDM-1 producers. J Clin Microbiol. 2011; 49:718-21. http://dx.doi/10.1128/JCM.01773-10

17. Queenan AM, Bush K. Carbapenemases: The versatile $\beta$-lactamases. Clin Microbiol Rev. 2007;20:440-58. http://dx. doi:10.1128/CMR.00001-07

18. Villegas MV, Lolans $\mathrm{K}$, del Rosario Olivera M, Suárez $\mathbf{C J}$, Correa A, Queenan AM, et al. First detection of metallo-betalactamase VIM-2 in Pseudomonas aeruginosa isolates from Colombia. Antimicrob Agents Chemother. 2006;50:226-9. http://dx.doi/10.1128/AAC.50.1.226-229.2006

19. Cuzon G, Naas T, Villegas MV, Correa A, Quinn JP, Nordmann P. Wide dissemination of Pseudomonas aeruginosa producing beta-lactamase blaKPC-2 gene in Colombia. Antimicrob Agents Chemother. 2011;55:5350-3. http://dx.doi/10.1128/AAC.00297-11

20. Yong D, Lee $\mathbf{Y}$, Jeong SH, Lee K, Chong Y. Evaluation of double-disk potentiation and disk potentiation tests using dipicolinic acid for detection of metallo- $\beta$-lactamaseproducing Pseudomonas spp. and Acinetobacter spp. J Clin Microbiol. 2012;50:3227-32. http://dx.doi/10.1128/JCM. 00818-12

21. Carvalhaes CG, Cayô R, Assis DM, Martins ER, Juliano L, Juliano MA, et al. Detection of SPM-1-producing Pseudomonas aeruginosa and class D $\beta$-lactamaseproducing Acinetobacter baumannii isolates by use of liquid chromatography-mass spectrometry and matrix-assisted laser desorption ionization-time of flight mass spectrometry. $\mathrm{J}$ Clin Microbiol. 2013;51:287-90. http://dx.doi/10.1128/JCM. 02365-12

22. Bonnin RA, Naas T, Poirel L, Nordmann P. Phenotypic, biochemical, and molecular techniques for detection of metallo- $\beta$-lactamase NDM in Acinetobacter baumannii. $\mathrm{J}$ Clin Microbiol. 2012;50:1419-21. http://dx.doi/10.1128/JCM. 06276-11

23. Correa A, Montealegre MC, Mojica MF, Maya JJ, Rojas LJ, De La Cadena EP, et al. First report of a Pseudomonas aeruginosa isolate coharboring KPC and VIM carbapenemases. Antimicrob Agents Chemother. 2012; 56:5422-3. http://dx.doi/10.1128/AAC.00695-12 